\title{
Upregulation of E-cadherin expression mediated by a novel dsRNA suppresses the growth and metastasis of bladder cancer cells by inhibiting $\beta$-catenin/TCF target genes
}

\author{
CHUANCHANG LI ${ }^{1}$, JIAXUAN LIU ${ }^{2}$, QINGSONG ZHANG ${ }^{1}$, KAI CUI ${ }^{1}$, \\ QIANGQIANG GE ${ }^{1,3}$, CHENGHE WANG $^{1,4}$ and ZHONG CHEN ${ }^{1}$
}

\begin{abstract}
${ }^{1}$ Department of Urology, Tongji Hospital, Tongji Medical College, Huazhong University of Science and Technology, Wuhan, Hubei 430030; ${ }^{2}$ Department of Pathology, Changzheng Hospital, The Second Military Medical University, Shanghai 200003;

${ }^{3}$ Department of Urology, Zhongnan Hospital, Wuhan University, Wuhan, Hubei 430071; ${ }^{4}$ Department of Urology, Ruijin Hospital, School of Medicine, Shanghai Jiaotong University, Shanghai 200025, P.R. China
\end{abstract}

Received November 11, 2017; Accepted March 21, 2018

DOI: $10.3892 /$ ijo.2018.4346

\begin{abstract}
Low expression levels of E-cadherin are correlated with poor prognosis in patients with bladder cancer (BCa). A small activating RNA (saRNA) targeting a specific promoter region can activate gene expression. In the present study, two small double-stranded RNAs (dsRNAs) targeting the promoter region of human E-cadherin were designed and synthesized, and the regulatory role of saRNAs in E-cadherin expression was investigated. The results of reverse transcription-quantitative polymerase chain reaction and western blotting demonstrated that transfection of dsEcad-346 into the BCa cell lines T24 and 5637 significantly activated E-cadherin expression. Furthermore, transfection of dsEcad-346 and miR-373 induced cell cycle arrest in G0/G1 phase, promoted apoptosis and significantly inhibited migration and invasion of $\mathrm{BCa}$ cells. Results of immunofluorescence and western blotting indicated that $\beta$-catenin was redistributed from the nucleus to the cell membrane following transfection of dsEcad-346 and miR-373. Additionally, the expression of $\beta$-catenin/T-cell factor complex (TCF) target genes (c-MYC, matrix metallopeptidase 2, cyclin D1) was suppressed following transfection of BCa cells with saRNA. Silencing of E-cadherin expression blocked the inhibitory effect of dsEcad-346 and miR-373 on BCa cells. In conclusion, a novel designed dsEcad-346 can activate the expression of E-cadherin in BCa cells. saRNA-mediated activation of E-cadherin expression inhibited the growth and metastasis of $\mathrm{BCa}$ cells by promoting the redistribution of
\end{abstract}

Correspondence to: Professor Zhong Chen, Department of Urology, Tongji Hospital, Tongji Medical College, Huazhong University of Science and Technology, 1,095 Jie Fang Avenue, Wuhan, Hubei 430030, P.R. China

E-mail: chenzhongtongji@126.com

Key words: RNA activation, E-cadherin, $\beta$-catenin, bladder cancer, migration and invasion, growth $\beta$-catenin from nucleus to cell membrane and inhibiting the $\beta$-catenin/TCF target genes.

\section{Introduction}

Bladder cancer ( $\mathrm{BCa})$ is the most common malignant tumour of the urinary system. It is the seventh most common cancer in men (1). In the United States, 79,030 newly diagnosed cases (60,490 male and 18,540 female) and 16,870 mortalities (12,240 male and 4,630 female) were reported in 2017 (2). Although $75 \%$ of newly diagnosed BCa present as non-muscleinvasive tumours, $\sim 30 \%$ of BCa cases managed by surgery still recur and progress to locally invasive or metastatic stages (3). Auxiliary chemotherapy is usually used to delay the recurrence and to prolong survival, however only half of the patients with muscle invasive BCa respond to adjuvant chemotherapy (4). The lack of effective treatment for the progression of $\mathrm{BCa}$ means there is an urgent need for studies on novel targets for gene therapy.

The epithelial cell-cell adhesion molecule cadherin 1, also termed epithelial cadherin (E-cadherin), is a well-known growth and invasion suppressor (5). Downregulation of E-cadherin is closely associated with tumour invasiveness, metastasis, and poor patient prognosis in various cancer types, including $\mathrm{BCa}(6,7)$. The majority E-cadherin and $\beta$-catenin protein is present as an E-cadherin/catenin adhesion complex located in cell-cell adherent junctions at the cell membrane; however, if not bound to surface E-cadherin, $\beta$-catenin becomes free to translocate to the nucleus where it transcriptionally activates several pro-migratory genes necessary for epithelial-mesenchymal transition (EMT) in association with the TCF/lymphoid enhancer-binding factor (LEF) transcription factor. Increasing E-cadherin/ $\beta$-catenin complex formation suppresses EMT and cell migration $(8,9)$. Several studies have reported that the recovery of E-cadherin expression, a tumour suppressor gene, can inhibit tumour progression, invasion and metastasis (10-12).

A small activating RNA (saRNA) targeting a particular promoter region of a gene can stimulate gene expression at the 
transcriptional level, a phenomenon known as RNA activation (RNAa) (13). Growing evidence has indicated that stimulating the expression of tumour suppressor genes using double stranded RNAs (dsRNAs) or microRNAs (miRNAs/miRs) has antitumour effects on various cancer cell types $(14,15)$. It has been demonstrated that miR-373 can activate E-cadherin expression in prostate cancer cells (16). Restoration of E-cadherin expression at the transcriptional level by RNAa may be a potentially effective therapy for cancer.

In the present study, a novel dsRNA was designed (dsEcad-346) and it was demonstrated that targeting the gene promoter region with the dsRNA significantly enhanced E-cadherin expression. In addition, the results demonstrated that transfection of the newly designed dsEcad-346 and miR-373 can inhibit the growth and metastasis of bladder cancer cells. Mechanistic research demonstrated that dsEcad-346 and miR-373 promote the transfer of $\beta$-catenin from the nucleus to the cell membrane via activating the expression of E-cadherin. With the reduction of $\beta$-catenin in the nucleus, the expression of $\beta$-catenin/T-cell factor complex (TCF) target genes c-MYC, matrix metallopeptidase 2 (MMP2), and cyclin D1 was inhibited.

\section{Materials and methods}

dsRNA design and saRNA synthesis. The promoter sequence $(1 \mathrm{~kb})$ of the E-cadherin gene was retrieved and scanned for dsRNA targets based on the rational dsRNA design rules (17). A BLAST search was performed to exclude those targets that shared homology with nonspecific human sequences (blast. ncbi.nlm.nih.gov/Blast.cgi) and to reject those targets with single nucleotide polymorphisms (genome.ucsc.edu/cgi-bin/ hgBlat). All the saRNA mimics were synthesized by RiboBio Co., Ltd. (Guangzhou, China). Control dsRNA (dsControl) was specifically designed to have no homology to any known human sequences (13). A small interfering RNA (siRNA) that specifically targets human E-cadherin mRNA (siEcad) was designed to silence E-cadherin expression (18). All the custom saRNA sequences are listed in Table I.

Cell culture and transfection. The human BCa 5637 and T24 cell lines were purchased from American Type Culture Collection (Manassas, VA, USA). The BCa cells were maintained in RPMI-1640 or $\alpha$-MEM (HyClone; GE Healthcare Life Sciences, Logan, UT USA) supplemented with $10 \%$ foetal bovine serum (Gibco; Thermo Fisher Scientific, Inc., Waltham, MA, USA) and $2 \mathrm{mM} / 1 \mathrm{~L}$-glutamine in a humidified atmosphere containing $5 \% \mathrm{CO}_{2}$ at $37^{\circ} \mathrm{C}$. Cells were seeded in growth medium without antibiotics at a density of $\sim 40 \%$ and then transfected with $50 \mathrm{nM}$ dsRNAs or miR-373 mimics using Lipofectamine RNAiMax (Invitrogen; Thermo Fisher Scientific, Inc.) according to the manufacturer's protocol.

Reverse transcription-quantitative polymerase chain reaction (RT-qPCR). Total cellular RNA was extracted from cells that had been transfected for $72 \mathrm{~h}$ using the TRIzol reagent (Invitrogen; Thermo Fisher Scientific, Inc.). RNA (500 ng) was reverse transcribed by using the Takara RT kit (Takara Biotechnology Co., Ltd., Dalian, China). RT reaction conditions were $37^{\circ} \mathrm{C}$ for $15 \mathrm{~min}$ and $85^{\circ} \mathrm{C}$ for $5 \mathrm{sec}$, according to the manufacturer's recommendation. qPCR amplification was conducted using SYBR Premix Ex Taq ${ }^{\mathrm{TM}}$ II (Takara Biotechnology Co., Ltd.) according to the manufacturer's instructions on the ABI PRISM 7300 system (Applied Biosystems; Thermo Fisher Scientific, Inc.). All qPCR reactions consisted of hot start activated step $\left(95^{\circ} \mathrm{C}\right.$ for $\left.30 \mathrm{sec}\right)$, and 40 cycles of $5 \mathrm{sec}$ at $95^{\circ} \mathrm{C}$ and $30 \mathrm{sec}$ at $60^{\circ} \mathrm{C}$. Endogenous gene expression was normalized to GAPDH levels in the cells. Each measure was done in triplicate and the change in expression of the target genes was quantified using the $2^{-\Delta \Delta C q}$ method (19). Specific primer sets were designed for qPCR and synthesized by Invitrogen (Thermo Fisher Scientific, Inc.; Table II).

Protein extraction and western blot analysis. Briefly, cells were harvested $72 \mathrm{~h}$ after dsRNA or miRNA treatment and lysed in ice-cold NP40 (Beyotime Institute of Biotechnology, Haimen, China) lysis buffer containing the protease inhibitor phenylmethanesulfonylfluoride (Roche Diagnostics, Basel, Switzerland) to obtain the total cellular protein. Nuclear and cytoplasmic $\beta$-catenin proteins were isolated from cells using a cell fractionation kit (Wuhan Boster Biological Technology, Ltd., Wuhan, China), according to the manufacturer's instructions. Protein concentration in lysates was quantified using an enhanced bicinchoninic acid assay kit (Beyotime Institute of Biotechnology) according to the manufacturer's instructions. Proteins $(40 \mu \mathrm{g})$ were separated using $8 \%$ SDS-PAGE and transferred onto polyvinylidene fluoride membranes (EMD Millipore, Billerica, MA, USA). Following blocking for $1 \mathrm{~h}$ at room temperature with $5 \%$ bovine serum albumin (Sigma-Aldrich; Merck KGaA, Darmstadt, Germany), they were incubated overnight at $4{ }^{\circ} \mathrm{C}$ with primary antibodies against E-cadherin (1:1,000; cat. no. 610181; BD Biosciences, San Jose, CA, USA), cyclin D1 (1:1,000; cat. no. AF0931; Affinity Biosciences, Cincinnati, OH, USA), c-MYC (1:1,000; cat. no. AF0358; Affinity Biosciences), MMP2 (1:1,000; cat. no. BM0569; Wuhan Boster Biological Technology, Ltd.), and $\beta$-catenin (1:1,000; cat. no. AF6266; Affinity Biosciences). $\beta$-actin (cat. no. BM0627; Wuhan Boster Biological Technology, Ltd.) was used as an internal control at a 1:500 dilution. Subsequently, the membranes were incubated with horseradish peroxidase-labelled secondary antibodies (1:5,000; cat. nos. S0001 and S0002; Affinity Biosciences) at room temperature for $2 \mathrm{~h}$. Antigen-antibody complexes were visualized using an enhanced chemiluminescence assay kit (Clarity Max Western ECL Substrate; Bio-Rad Laboratories, Inc., Hercules, CA, USA) for protein detection. The ECL signals were detected by chemiluminescence imaging system (GeneGnome XRQ; Syngene Europe, Cambridge, UK). The expression of $\beta$-catenin in the nuclear and cytoplasmic compartments was determined as described above.

Wound-healing assay. To evaluate the effects of the overexpression of dsEcad-346 and miR-373 on the migratory ability of BCa cells, a scratch assay was performed. Cells were transfected with saRNAs or the negative control sequence for $72 \mathrm{~h}$. Then, $\sim 5 \times 10^{5}$ cells were reseeded in a new 6 -well plate. When the cells had grown to confluence, a micropipette tip was used to create a cell monolayer. The scratch widths at $0 \mathrm{~h}, 12 \mathrm{~h}$ and 
Table I. Sequences of synthesized dsRNA and microRNA.

\begin{tabular}{ll} 
Synthesized RNAs & Sequences (5'-3') \\
\hline dsEcad-238 (S) & GGATTCGAACCCAGTGGAA[dT][dT] \\
dsEcad-238 (AS) & UUCCACUGGGUUCGAAUCC[dT][dT] \\
dsEcad-346 (S) & ACCCCATCTCCAAAACGAA[dT][dT] \\
dsEcad-346 (AS) & UUCGUUUUGGAGAUGGGGU[dT][dT] \\
microRNA-373 & ACUCAAAAUGGGGGCGCUUUCC \\
dsControl (S) & ACUACUGAGUGACAGUAGA[dT][dT] \\
dsControl (AS) & UCUACUGUCACUCAGUAGU[dT][dT] \\
siEcad (S) & GGCCTGAAGTGACTCGTAA \\
siEcad (AS) & CCGGACUUCACUGAGCAUU
\end{tabular}

ds, double stranded RNA; Ecad, E-cadherin; si, small interfering RNA.

$24 \mathrm{~h}$ after wounding were calculated from photomicrographs captured using an inverted microscope (Olympus Corporation, Tokyo, Japan).

Transwell assays. The motility of cells was evaluated with a 24 -well Boyden chamber with an $8-\mu \mathrm{m}$ pore size polycarbonate membrane (Corning Incorporated, Corning, NY, USA). For the Transwell invasion assays, the membrane was pre-coated with $50 \mu 133.3 \%$ Matrigel (BD Bioscience) to form a matrix barrier. Following transfection of candidate saRNAs for $72 \mathrm{~h}, 200 \mu \mathrm{l}$ serum-free medium containing $5 \times 10^{4}$ cells were seeded in the upper chamber. A total of $500 \mu \mathrm{l}$ of medium with $10 \%$ FBS was added to the lower chamber as a chemoattractant. After incubation for $48 \mathrm{~h}$, the cells were fixed with $4 \%$ formaldehyde for $10 \mathrm{~min}$ and stained with $0.1 \%$ crystal violet at room temperature for $15 \mathrm{~min}$. Non-migrated cells in the upper chamber were removed and the cells that had invaded the lower surface of the membrane were images using an inverted microscope (Olympus Corporation), and the number of cells was counted in four randomized visual fields. The Transwell migration assay was performed using the same method as the invasion assay, except the upper chambers were Matrigel-free.

Cell cycle analysis and evaluation of apoptosis by flow cytometry. To assess the distribution of cells in different phases of the cell cycle, a flow cytometric analysis of the cell cycle distribution was performed. After transfection of the corresponding miRNA or dsRNA for $72 \mathrm{~h}$, the cells were harvested and fixed with $70 \%$ cold ethanol overnight. Then, the cells were incubated with RNase A at $37^{\circ} \mathrm{C}$ for $30 \mathrm{~min}$ and resuspended in propidium iodide (PI; Cell Cycle Detection kit; Nanjing KeyGen Biotech Co., Ltd., Nanjing, China) at $4^{\circ} \mathrm{C}$ for $30 \mathrm{~min}$. Then, the samples were analysed for PI fluorescence intensity by flow cytometry to assess the relative cellular DNA content, and the data were interpreted using CellQuest software (version 3.3; BD Biosciences). Apoptosis was measured by flow cytometry following staining of cells with Annexin V-fluorescein isothiocyanate (FITC) and PI in accordance with manufacturer's recommendations [Multisciences (Lianke) Biotech Co., Ltd., Hangzhou, China]. The cell apoptosis rate was calculated as
Table II. Primer sequences used in this study.

\begin{tabular}{ll}
\hline Name & \multicolumn{1}{c}{ Sequences (5'-3') } \\
\hline E-cadherin (F) & ACCAGAATAAAGACCAAGTGACCA \\
E-cadherin (R) & AGCAAGAGCAGCAGAATCAGAAT \\
GAPDH (F) & TCCCATCACCATCTTCCA \\
GAPDH (R) & CATCACGCCACAGTTTCC \\
c-MYC (F) & CTGCTTAGACGCTGGATTT \\
c-MYC (R) & TCGTAGTCGAGGTCATAGTTC \\
MMP2 (F) & ATAACCCACCTAGACCCTAAA \\
MMP2 (R) & GGAGTCCGTCCTTACCGTCA \\
Cyclin D1 (F) & GCTGCGAAGTGGAAACCATC \\
Cyclin D1 (R) & CCTCCTTCTGCACACATTTGAA
\end{tabular}

F, forward; R, reverse; MMP2, matrix metallopeptidase 2.

follows: Cell apoptosis rate $(\%)=($ apoptotic cell number $/$ total cell number) $\mathrm{x} 100$.

Immunofluorescence. The localization of E-cadherin and $\beta$-catenin in cells was detected by immunofluorescence. At $72 \mathrm{~h}$ post-transfection, cells were fixed in $4 \% \mathrm{CH} 3 \mathrm{OH}$ for $10 \mathrm{~min}$ at room temperature and then permeabilised with $0.5 \%$ Triton X-100 (Sigma-Aldrich; Merck KGaA) at room temperature for $20 \mathrm{~min}$. Following blocking with $3 \%(\mathrm{w} / \mathrm{v})$ bovine serum albumin for $1 \mathrm{~h}$ at $25^{\circ} \mathrm{C}$, the cells were incubated with the E-cadherin antibody (1:100; cat. no. 610181; $\mathrm{BD}$, Biosciences) and the $\beta$-catenin antibody $(1: 100$; cat. no. AF6266; Affinity Biosciences) overnight. FITCconjugated IgG (1:50; Wuhan Boster Biological Technology, Ltd.) was used as the secondary antibody. Nuclei were counterstained with DAPI $(0.05 \mathrm{mg} / \mathrm{ml})$ for $5 \mathrm{~min}$ at room temperature. Subsequently, the samples were analysed with an Olympus inverted fluorescence microscope (Olympus Corporation).

Statistical analysis. All data are presented as the mean \pm standard deviation. Statistical analyses were performed by SPSS version 13.0 software (SPSS Inc., Chicago, IL, USA) and GraphPad Prism 5.0 software (GraphPad Software, Inc., La Jolla, CA, USA). The differences between two groups were analysed using two-tail Student's t-test and differences between more than two groups were analysed using one-way analysis of variance (post hoc test, Tukey). $\mathrm{P}<0.05$ was considered to indicate a statistically significant difference.

\section{Results}

dsRNA targeting the promoter of E-cadherin activates gene expression in BCa cells. E-cadherin expression is reduced in $\mathrm{BCa}$ cells and that reduced expression is associated with cancer progression and poor overall survival of patients with $\mathrm{BCa}(7,11)$. To enhance the expression of E-cadherin in $\mathrm{BCa}$ cells, two 21-nucleotide dsRNAs targeting the E-cadherin promoter at sequence positions -238 (dsEcad-238) and -346 (dsEcad-346) were designed and synthesized (Fig. 1A). The 
A



dsEcad-238:

5'-3' GGATTCGAACCCAGTGGAA[dT][dT]

|||||||||||||||||||

[dT][dT]CCUAAGCUUGGGUCACCUU 3 '-5'

\author{
dsEcad-346: \\ 5'-3' ACCCCATCTCCAAAACGAA $[\mathrm{dT}][\mathrm{dT}]$ \\ ||||||||||||||||||| \\ [dT]|dT]UGGGGUAGAGGUUUUGCUU $3{ }^{\prime}-5$
}

B


C

T24

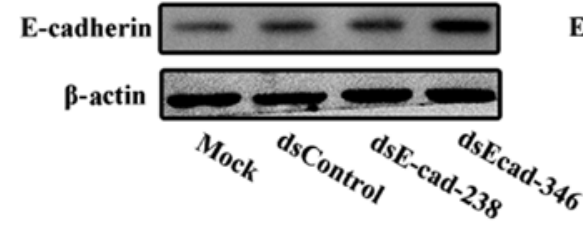

5637

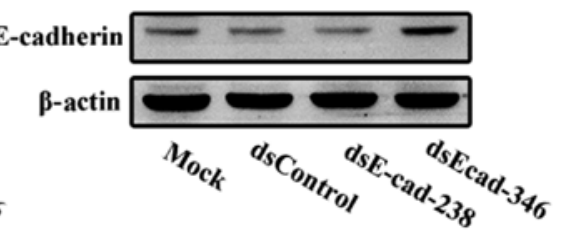

Figure 1. dsEcad-346 enhances E-cadherin expression in BCa cells. (A) Schematic representation of the E-cadherin promoter and the corresponding dsEcad-238 and dsEcad-346 target sites, and the complementary seed sequences. (B) BCa cells (T24 and 5637) were transfected with the indicated dsRNAs (50 nM) for $72 \mathrm{~h}$. Mock samples were transfected in the absence of small activating RNA. The expression of E-cadherin mRNA was estimated by reverse transcriptionquantitative polymerase chain reaction. (C) Immunoblot analysis was performed to measure the protein expression of E-cadherin. $\beta$-actin served as an internal control. ${ }^{* * *} \mathrm{P}<0.001$ vs. Mock; ${ }^{\# \# *} \mathrm{P}<0.001$ vs. dsControl. BCa, bladder cancer; ds, double stranded RNA; Ecad, E-cadherin.

candidate dsRNAs were transfected into human BCa cell lines for $72 \mathrm{~h}$. The mRNA and protein expression levels of E-cadherin were detected by RT-qPCR and western blotting, respectively. E-cadherin mRNA levels in dsEcad-346-treated T24 and 5637 cells were significantly elevated by $\sim 8.3-$ and $\sim 3.7$-fold, respectively, compared with the levels in mock and dsControl transfected cells. However, there was no significant change in the dsEcad-238-transfected group (Fig. 1B). Upregulation of the E-cadherin mRNA was strongly associated with an increase in E-cadherin protein expression (Fig. 1C). These results suggest that the novel designed dsEcad-346 induces E-cadherin expression by targeting the E-cadherin gene promoter in $\mathrm{BCa}$ cells.

dsEcad-346 and miR-373 enhance the expression of E-cadherin on the surface of the cell membrane and inhibit the proliferation of BCa cells. To further detect the effect of saRNAs on the activation of E-cadherin, the expression of E-cadherin was examined using immunofluorescence. The expression of E-cadherin was increased on the membrane surfaces of T24 and 5637 cells transfected with dsEcad-346 (Fig. 2A). In addition, the results show that miR-373 can also upregulate the expression of E-cadherin on the cell membrane surface of T24 and 5637 cells, this is similar to the results of previous experiments in our experimental group that miRNA-373 can activate E-cadherin expression in bladder tumour cells (20). E-cadherin is known to inhibit tumourigenicity and tumour transmission. Previous research demonstrated that upregulation of the expression of E-cadherin induces apoptosis and inhibits the proliferation of breast carcinoma cells in vitro and in vivo (21). To further evaluate the physiological effects of dsEcad-346 and miR-373 on BCa cell growth, flow cytometry was performed to assess the distribution of cells in the cell cycle. Compared with the dsControl group, the dsEcad-346and miR-373-transfected cells demonstrated a marked accumulation in the G0/G1 phase and a decrease in the $\mathrm{S}$ and $\mathrm{M}$ phases (Fig. 2B).

To analyse the cell apoptosis rate induced by the upregulation of E-cadherin expression, dsEcad-346 and miR-373 were transfected into T24 and 5637 cells for $72 \mathrm{~h}$, and the cell apoptosis rates were analysed by flow cytometry assay. As shown in Fig. 2C and D, overexpression of dsEcad-346 and miR-373 increased apoptosis in T24 and 5637 cells. The results indicated that transfection of dsEcad-346 and miR-373 blocked 
A
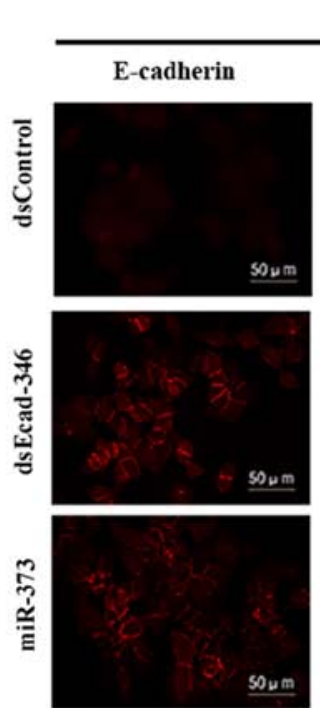

B

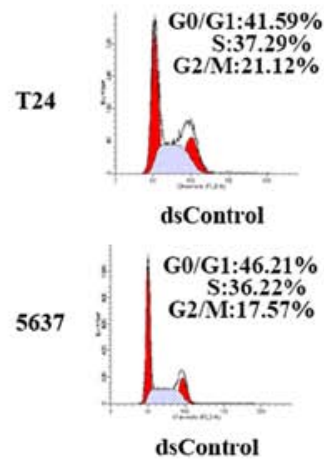

T24
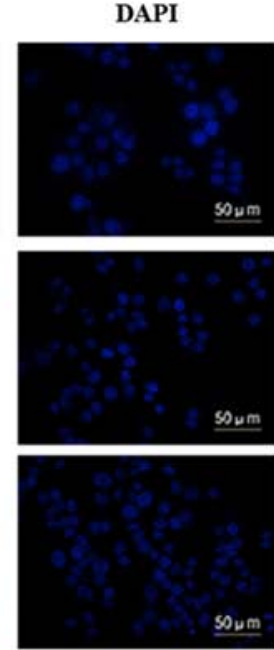

SOp
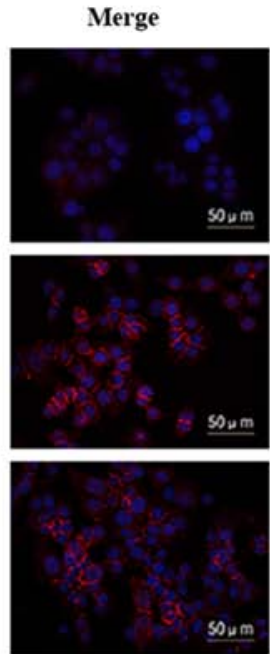

Soum
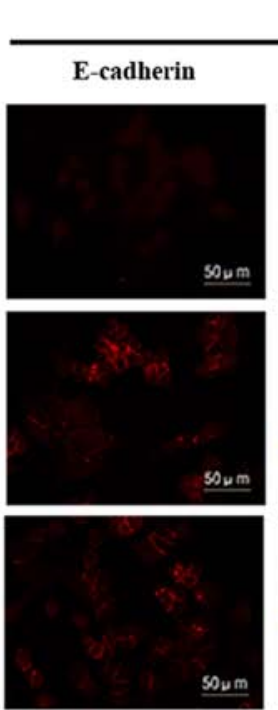

Soum
5637
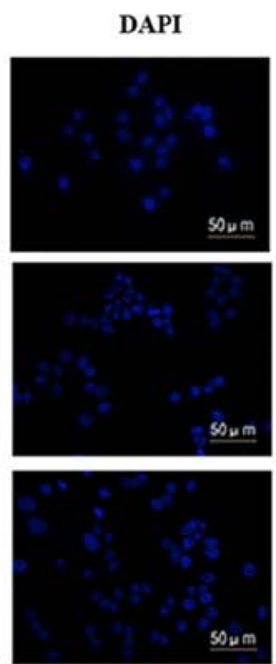

Soum
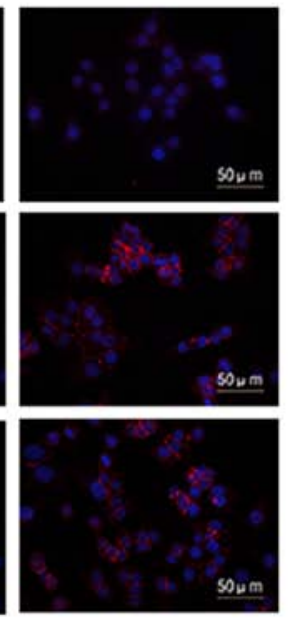

C
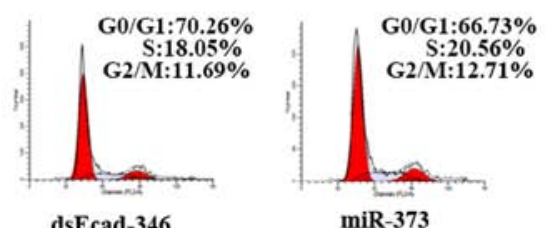

dsEcad-346
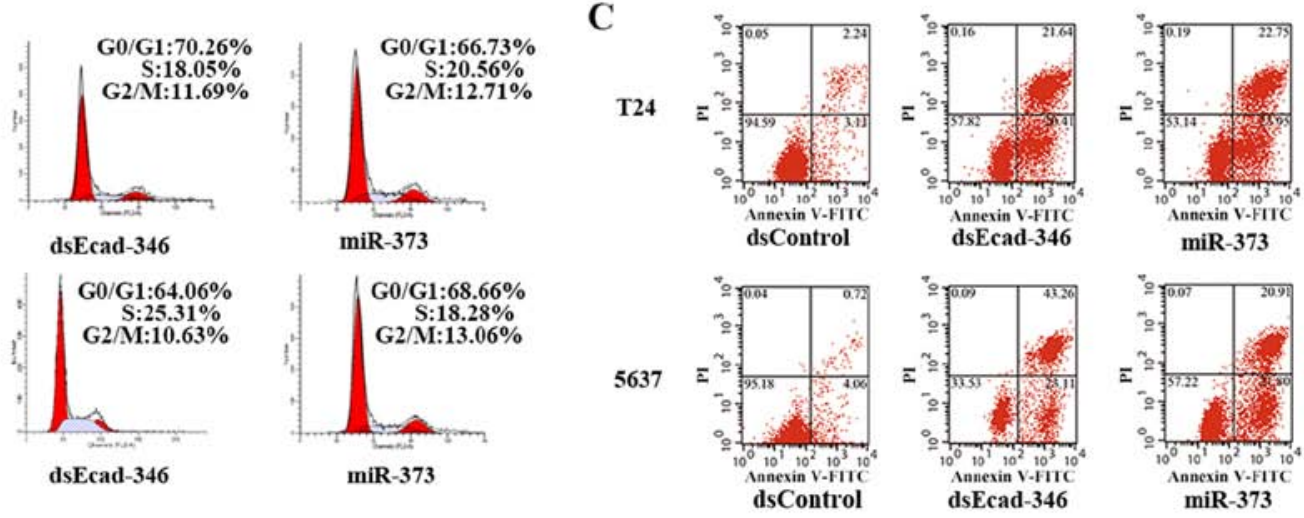

D

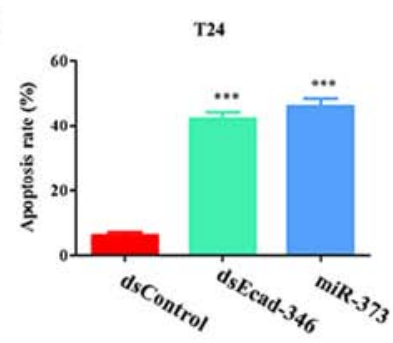



Figure 2. dsEcad-346 and miR-373 enhance the expression of E-cadherin on the surface of the cell membrane and inhibited the proliferation of bladder cancer cells. T24 and 5637 cells were transfected with $50 \mathrm{nM}$ dsControl, dsEcad-346 or miR-373 for $72 \mathrm{~h}$. (A) Expression of E-cadherin (red) in BCa cells was detected by immunofluorescence. The merged images represent overlays of E-cadherin (red) and nuclear staining by DAPI (blue). Scale bar, $50 \mu \mathrm{m}$. (B) Cell cycle distribution was determined by flow cytometry. The \% values are representative one experiment. (C) Flow cytometry analysis of cell apoptosis rates in BCa cells transfected with dsEcad-346, miR-373 or dsControl. (D) Percentage of apoptotic cells. " $\mathrm{P}<0.001$ vs. dsControl. BCa, bladder cancer; ds, double stranded RNA; Ecad, E-cadherin; miR, microRNA; PI, propidium iodide; FITC, fluorescein isothiocyanate.

cell cycle progression in the G0/G1 phase, which promoted the apoptosis of cells.

dsEcad-346 and miR-373 inhibit migration and invasion of BCa cells. To determine the effects of saRNAs on the migration and invasion capacity of BCa cells, wound healing and Transwell migration assays were performed. In the wound healing assay, the migration distances of the cells transfected with dsEcad-346 and miR-373 were significantly reduced compared with the dsControl group after $12 \mathrm{~h}$ and $24 \mathrm{~h}$ of incubation (Fig. 3A and B). The results of the Transwell assay revealed that dsEcad-346 and miR-373 treatment resulted in a significant decrease in motility and invasion compared with the dsControl group (Fig. 3C and D).

dsEcad-346 and miR-373 redistribute $\beta$-catenin from the nucleus to the cell membrane, and inhibit $\beta$-catenin/TCF target genes in BCa cells. To understand the mechanism of the inhibition of growth and migration by saRNAs through the upregulation of the expression of E-cadherin, the expression of $\beta$-catenin/TCF target genes were examined. To address this issue, RT-qPCR and western blotting were performed to determine the mRNA and protein expression levels of c-MYC, MMP2, and cyclin D1. RT-qPCR results demonstrated that transfection of dsEcad-346 
A

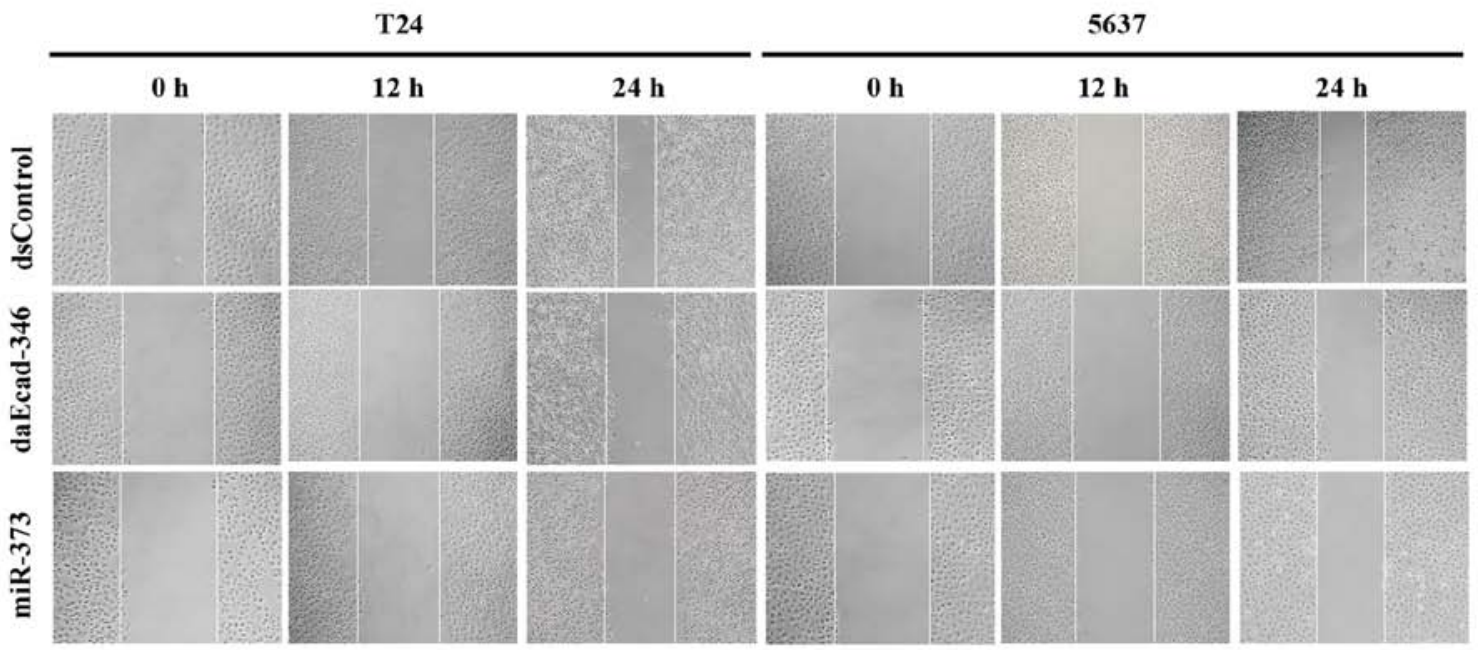

B
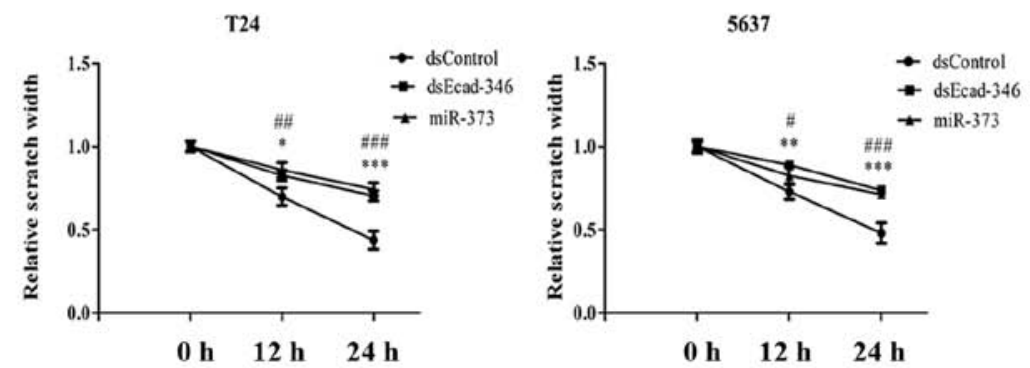

C


D
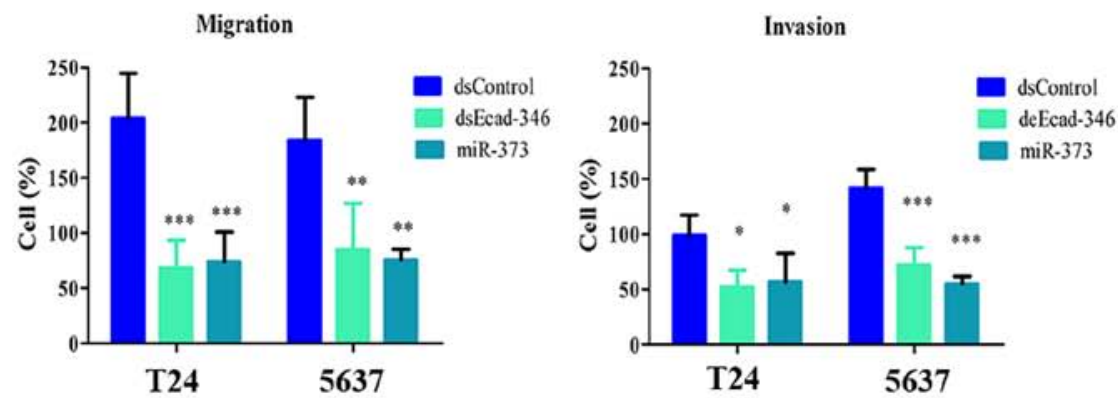

Figure 3. dsEcad-346 and miR-373 inhibit BCa cell migration and invasion. The effects of dsEcad-346 and miR-373 overexpression on migration and invasion in BCa cells were assessed by wound healing and Transwell assays. (A) Representative images and (B) quantification of the BCa cell migratory ability, as determined by the wound healing assay following transfection of dsEcad-346 and miR-373. (C) Representative pictures of the Transwell assay (x200). (D) The cells invading through the Matrigel and the membrane were counted in four random fields of each group. The results are presented as a per cent (\%) compared with the dsControl group (100 transmembrane cells per field represent $100 \%)$. ${ }^{*} \mathrm{P}<0.05,{ }^{* *} \mathrm{P}<0.01$ and ${ }^{* * *} \mathrm{P}<0.001$ vs. dsControl. BCa, bladder cancer; ds, double stranded RNA; Ecad, E-cadherin; miR, microRNA.

and miR-373 mimics significantly decreased c-MYC, MMP2 and cyclinD1 mRNA levels in T24 and 5637 cells (Fig. 4A and B). Western blotting further confirmed significant suppression of the protein expression of these genes (Fig. 4C and D).
To explore whether E-cadherin activation by saRNA can influence $\beta$-catenin status in $\mathrm{BCa}$ cells, $\beta$-catenin expression was evaluated by immunofluorescence and western blotting. Compared with that of the dsControl group, the abundance 
A
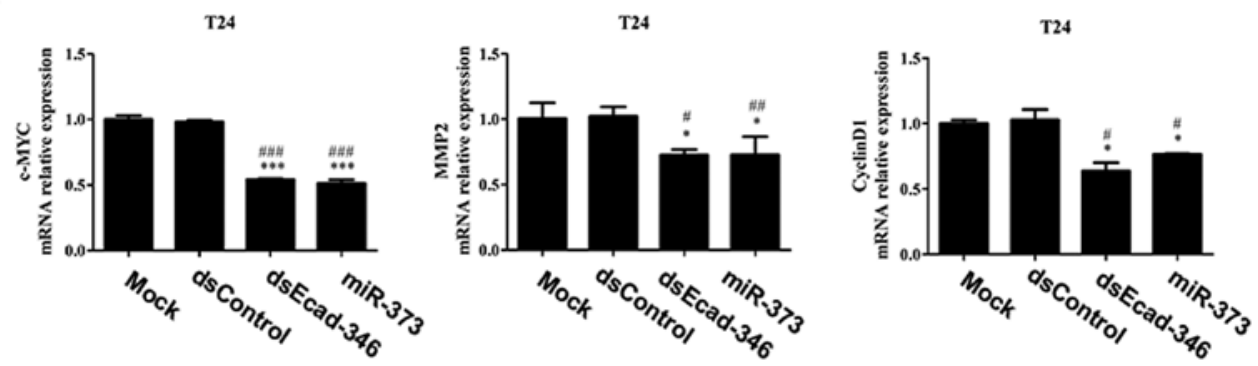

B

5637

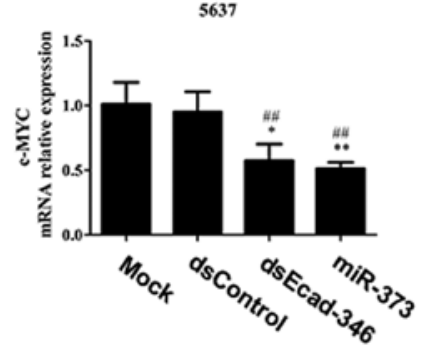

C

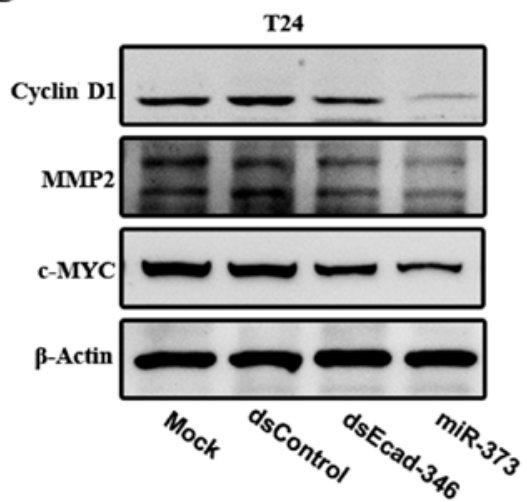

5637

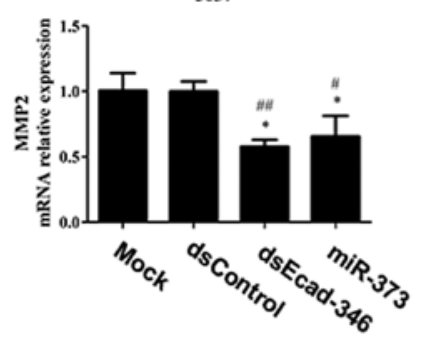

D

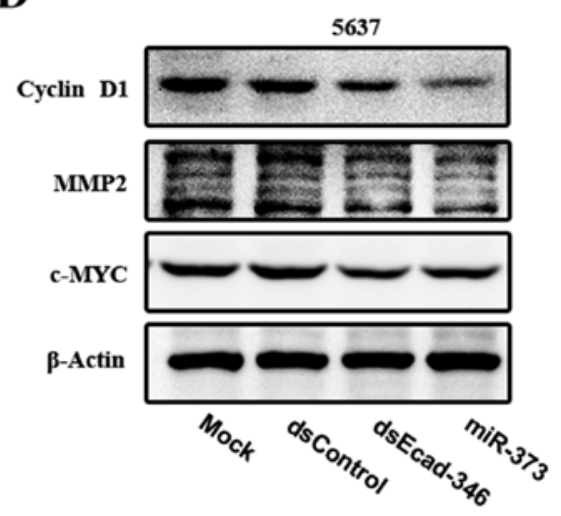

Figure 4. dsEcad-346 and miR-373 inhibit $\beta$-catenin/T-cell factor complex target genes in bladder cancer cells. Cells were transfected with 50 nM dsControl, dsEcad-346 or miR-373 for $72 \mathrm{~h}$. Relative expression levels of c-MYC, MMP2 and cyclin D1 mRNA (A) T24 cells and (B) 5637 cells were detected by reverse transcription-quantitative polymerase chain reaction. GAPDH served as a loading control. ${ }^{* * *} \mathrm{P}<0.001$ vs. Mock; ${ }^{\# \# \# P<0.001 ~ v s . ~ d s C o n t r o l . ~ c-M Y C, ~ M M P 2 ~}$ and cyclin D1 protein levels in (C) T24 and (D) 5637 cells were measured by western blotting. $\beta$-actin was used as an internal control. MMP2, matrix metallopeptidase 2; ds, double stranded RNA; Ecad, E-cadherin; miR, microRNA.

of plasma membrane $\beta$-catenin in T24 and 5637 cells treated with dsEcad-346 and miR-373 was significantly increased, and nuclear abundance of $\beta$-catenin was reduced (Fig. 5A). However, immunoblot analysis suggested that the change in the total level of $\beta$-catenin was not marked (Fig. 5B). Cytosolic and nuclear fractions of the cell lysates were isolated, and the transfection of dsEcad-346 and miR-373 resulted in an increased level of cytosolic $\beta$-catenin and a decreased level of nuclear $\beta$-catenin (Fig. 5B). These results suggest that saRNA redistributes $\beta$-catenin from the nucleus to the cell membrane, resulting in the inhibition of $\beta$-catenin/TCF target genes.

\section{dsEcad-346 and miR-373 modulate $\beta$-catenin/TCF target} genes via activation of E-cadherin expression. To demonstrate that dsEcad-346 and miR-373 supress the expression of $\beta$-catenin/TCF target genes via elevated expression of E-cadherin, siEcad was used to knockdown E-cadherin expression in T24 and 5637 cells. Following co-transfection dsEcad-346 or miR-373 with siEcad in BCa cells for $72 \mathrm{~h}$, the results demonstrated that the effect of E-cadherin activation mediated by the two saRNAs was markedly abrogated at the mRNA and protein levels (Fig. 6A and B). Then, the expression of c-MYC, MMP2 and cyclin D1 were examined in BCa cells. As illustrated, co-transfection with siEcad increased c-MYC, MMP2 and cyclin D1 expression dsEcad-346 or miR-373 (Fig. 6C and D). This result suggested that deEcad-346 and miR-373-induced inhibition of c-MYC, MMP2 and cyclin D1 expression is associated with expression of E-cadherin.

Depletion of the expression of $E$-cadherin reverses the effects of dsEcad-346 and miR-373 on the inhibition of the growth and metastasis of BCa cells. Whether activation of E-cadherin was involved in the inhibition of the growth and metastasis of BCa cells by dsEcad-346 or miR-373 was investigated. T24 and 5637 cells were co-transfected by dsEcad-346 or miR-373 with siEcad to silence the expression of E-cadherin. Flow cytometry revealed a marked reduction in the percentage of G0/G1 cells and corresponding increases in $\mathrm{S}$ and $\mathrm{G} 2 / \mathrm{M}$ populations in treatment groups co-transfected with siEcad compared with dsEcad-346 or miR-373 (Fig. 7A). These results indicate that 
A



B

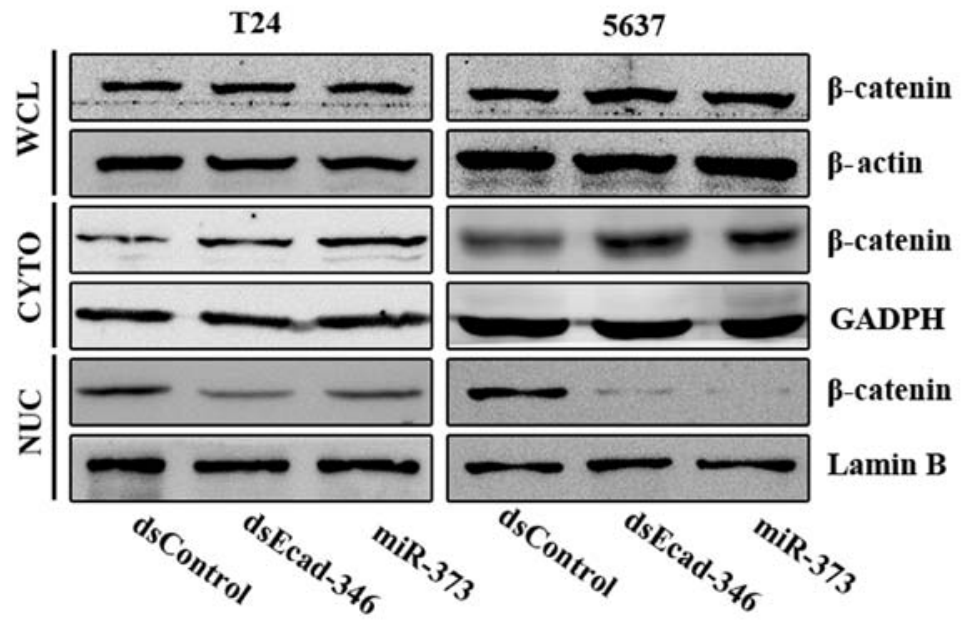

Figure 5. dsEcad-346 and miR-373 redistributed $\beta$-catenin from the nucleus to the cell membrane in BCa cells. (A) Immunofluorescence analysis of $\beta$-catenin (green) in the indicated BCa cells. The merged images represent overlays of $\beta$-catenin (green) and nuclear staining by DAPI (blue). Scale bar, $50 \mu \mathrm{m}$. (B) $\beta$-catenin expression in whole-cell lysates, cytoplasm and nuclear fractions as detected by western blotting. ds, double stranded RNA; Ecad, E-cadherin; miR, microRNA; WCL, whole-cell lysates; CYTO, cytoplasm; NUC, nuclear fraction.

silencing the expression of E-cadherin significantly reduced the cycle arrest that was mediated by saRNAs. Correspondingly, cell apoptosis was also decreased following the knockdown of E-cadherin (Fig. 6B and C). Additionally, the ability to inhibit cell migration and invasion mediated by dsEcad-346 and miR-373 was significantly reduced by depleting E-cadherin (Fig. 6D and E). Taken together, the findings indicated that the antitumour effect of dsEcad-346 and miR-373 in BCa cells is predominantly mediated via upregulation of E-cadherin expression.

\section{Discussion}

Reduced E-cadherin expression is associated with poor prognosis, and promotes the progression and metastasis of BCa (22). In the present study, a novel dsRNA, dsEca-346, was designed and it was demonstrated that dsEca-346 significantly induced E-cadherin expression in BCa cells. The results demonstrated that upregulation of E-cadherin by transcriptional activation (dsEcad-346 or miR-373) markedly inhibited cell migration and invasion, and induced cell cycle arrest and apoptosis of BCa cells. Mechanistically, upregulation of E-cadherin expression by saRNA resulted in decreased expression levels of c-MYC, MMP2 and cyclin D1. In addition, saRNA redistributed $\beta$-catenin from the nucleus to the cell membrane by promoting E-cadherin expression. Silencing the increased expression of E-cadherin using siRNA indicated that dsEcad-346 and miR-373 inhibited the growth and metastasis of BCa cells mainly through the activation of E-cadherin expression.

RNAa is a recently discovered mechanism of gene regulation that provides a new therapeutic tool for the treatment of cancer $(13,23)$. Our previous study demonstrated that endogenous miR-1236-3p and miR-370-5p induce p21 expression in bladder cancer cells. However, transfection of hepatocellular carcinoma cells with miR-1236-3p and miR-370-5p did not affect p21 expression (14). These results indicated that differences in promoter environments in different cancer cells may affect miRNA-induced gene activation. Furthermore, miRNAs often have several target genes, which may induce off-target effects. There is growing evidence that miR-373 can inhibit the expression of dickkopf WNT signalling pathway inhibitor 1, mechanistic target of rapamycin kinase and Rab22a member RAS oncogene family by targeting their 3'-untranslated regions and may have a role in tumour suppression or promotion in different tumours (24-26). Thus, it is necessary 
A

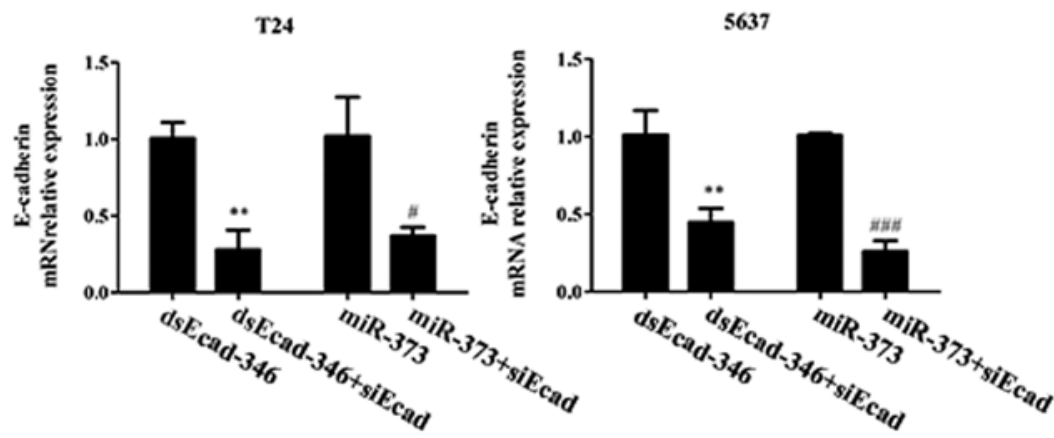

$\mathbf{C}$
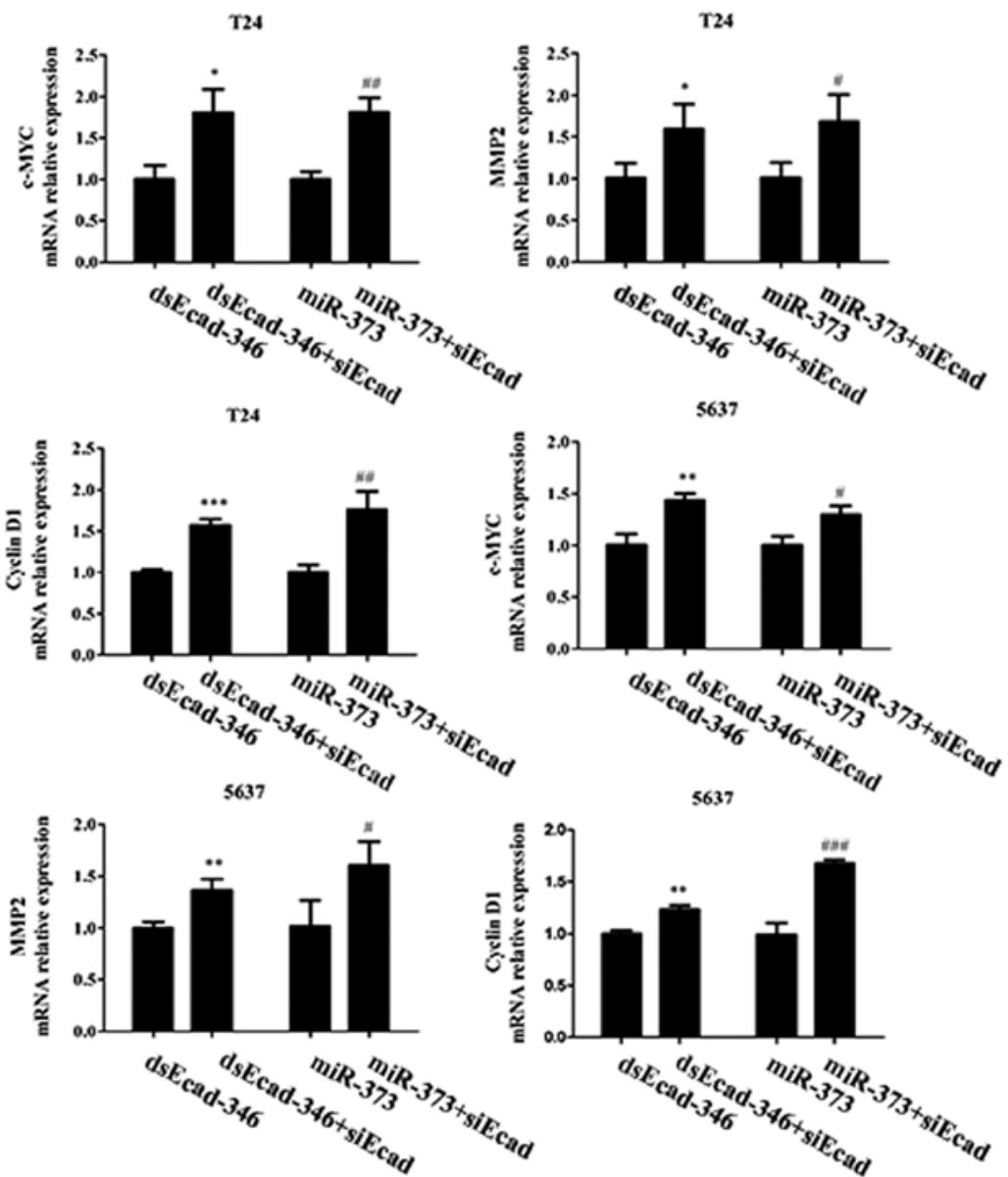

B

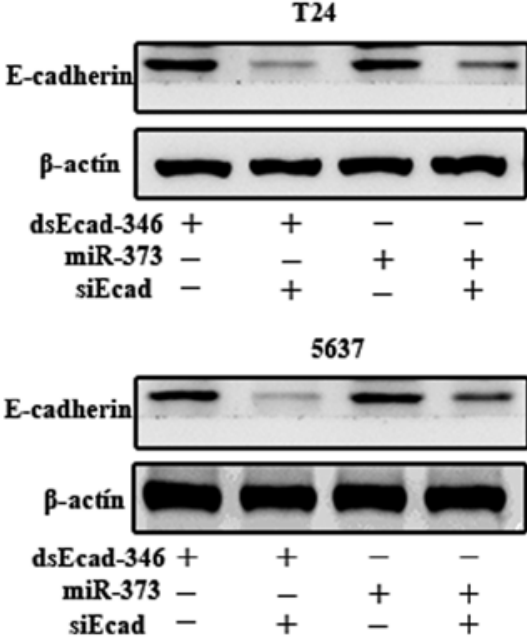

D
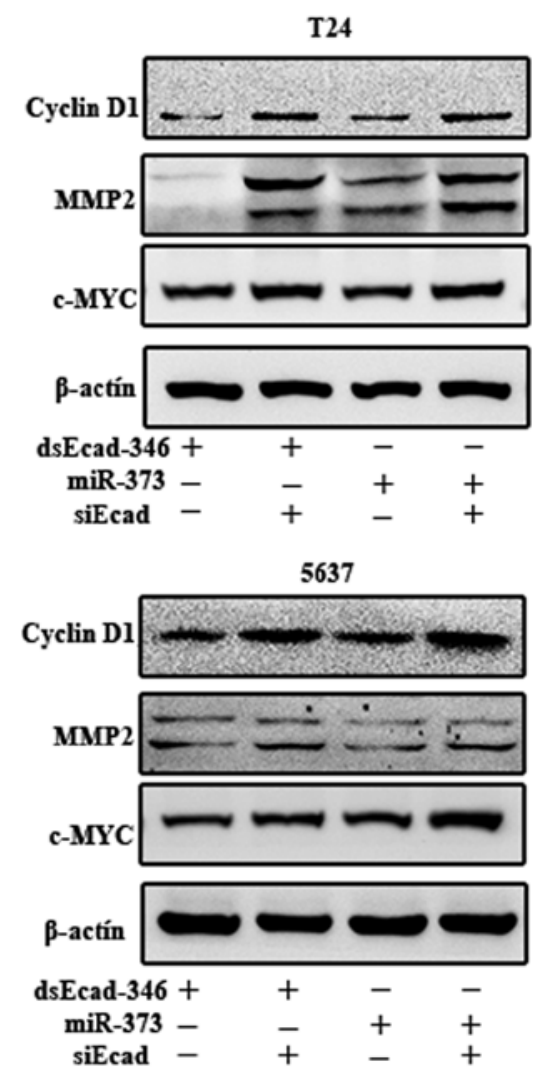

Figure 6. dsEcad-346 and miR-373 modulate $\beta$-catenin/T-cell factor complex target genes mainly by activating E-cadherin expression. (A) Expression levels of E-cadherin mRNA in T24 and 5637 cells co-transfected with the indicated saRNA and siEcad were measured by quantitative polymerase chain reaction. The values of E-cadherin were normalized to GAPDH. ${ }^{* *} \mathrm{P}<0.01$ vs. dsEcad-346; ${ }^{\#} \mathrm{P}<0.05$ and ${ }^{\# \# \#} \mathrm{P}<0.001$ vs. miR-373. (B) Protein expression levels of E-cadherin were detected by western blotting. $\beta$-actin served as an internal control. (C) Reverse transcription-quantitative polymerase chain reaction and (D) western blotting were used to detect the expression of c-MYC, MMP2 and cyclin D1 in T24 and 5637 cells co-transfected with the indicated saRNA and siEcad. ${ }^{*} \mathrm{P}<0.05,{ }^{* *} \mathrm{P}<0.01$ and ${ }^{* * *} \mathrm{P}<0.001$ vs. dsEcad-346; ${ }^{*} \mathrm{P}<0.05$, ${ }^{\# \#} \mathrm{P}<0.01$ and ${ }^{\# \# \#} \mathrm{P}<0.001$ vs. miR-373. saRNA, small activating RNA; ds, double stranded RNA; Ecad, E-cadherin; si, small interfering RNA; miR, microRNA; MMP2, matrix metallopeptidase 2.

to design specific on-target dsRNA sequences. Place et al (16) demonstrated that, unlike miR-373, which is highly complementary to E-cadherin and cold shock domain containing C2 (CSDC2) gene promoter sites and readily promotes the expression of both genes, dsEcad-215 and dsCSDC2-670 only enhance the expression of E-cadherin or CSDC2 specifically. Thus, synthetic dsRNAs seems more suitable for precisely targeted gene therapy than miRNAs. 
A



B


D

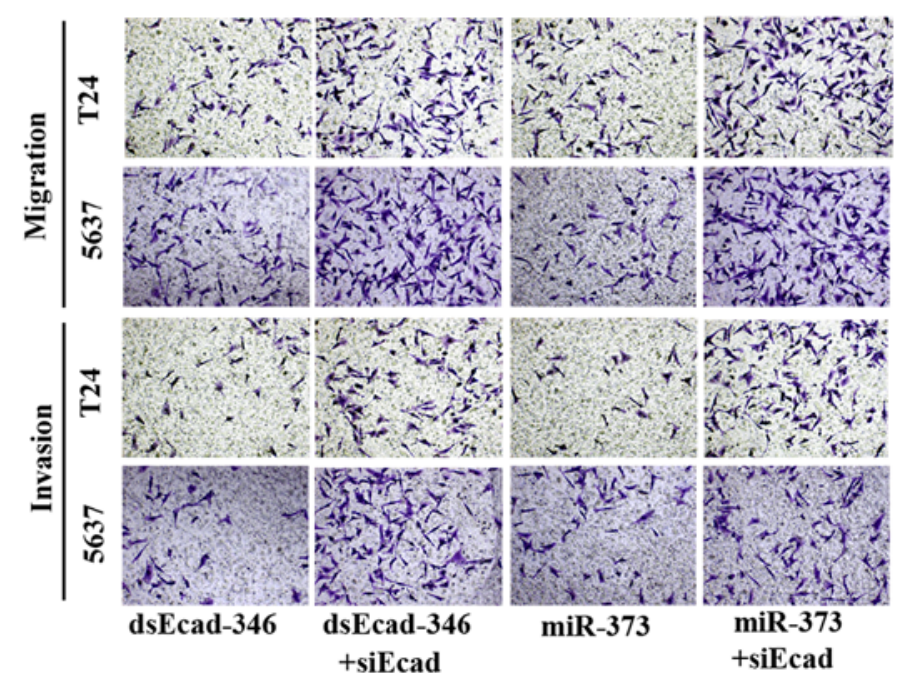

$\mathbf{E}$
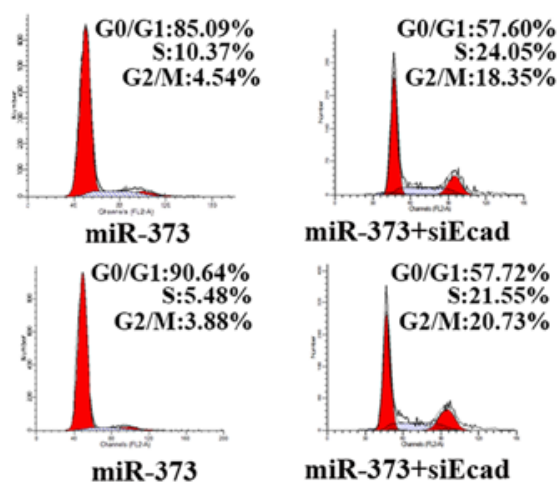

miR-373+siEcad

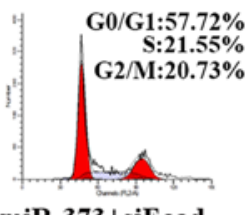

miR-373+siEcad

C
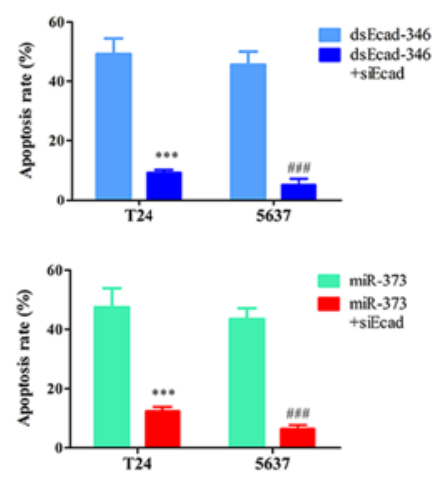
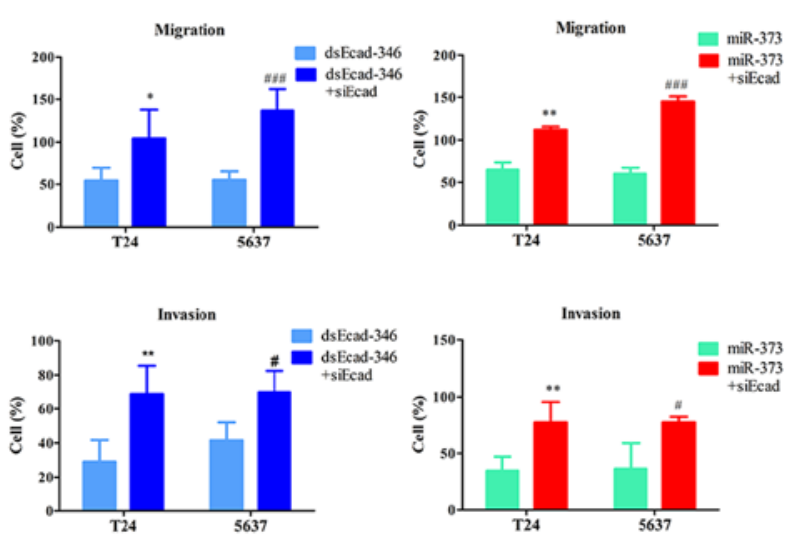

Figure 7. Depletion of E-cadherin expression reverses the effect of saRNA on the inhibition of the growth and metastasis of BCa cells. T24 and 5637 cells were co-transfected with the indicated saRNA and siEcad for $72 \mathrm{~h}$. (A) Cell cycle arrest induced by dsEcad-346 and miR-373 was blocked when they were co-transfected with siEcad. The \% values are representative one experiment. (B) Representative flow cytometry images of cell apoptosis. (C) Percentages of apoptotic cells. ${ }^{* * *} \mathrm{P}<0.001$ vs. dsEcad-346, ${ }^{\# \#} \mathrm{P}<0.001$ vs. miR-373. (D) Representative images of the Transwell migration and invasion assay for the indicated BCa cells (x200). (E) Numbers of migrated and invaded cells were quantified in four random images from each group. The results are presented as percentages (\%) relative to dsEcad-346 or miR-373 group (100 transmembrane cells per field represent $100 \%)$. ${ }^{*}<0.05$ and ${ }^{* *} \mathrm{P}<0.01$ compared to the dsEcad-346 group, ${ }^{\#} \mathrm{P}<0.05$ and ${ }^{\# \#} \mathrm{P}<0.001$ vs. miR-373. saRNA, small activating RNA; BCa, bladder cancer; ds, double stranded RNA; Ecad, E-cadherin; si, small interfering RNA; miR, microRNA; PI, propidium iodide; FITC, fluorescein isothiocyanate.

However, even well-selected dsRNA cannot avoid partial sequence homology to other coding and non-coding sequences (27). Thus, further research is required to identify whether dsRNA-regulated E-cadherin activation will induce miRNA-like mechanisms of post-transcriptional gene silencing. In this study, not every dsRNA tested activated E-cadherin expression. In addition, dsEcad-346 significantly activated E-cadherin expression in T24 cells ( $~ 8.3$-fold), whereas the activation effect in 5637 cells was weaker $(\sim 3.7$-fold). As previously reported, a dsRNA that works in one cell type may not work with equal efficacy in another (28). It is necessary to fully elucidate the mechanism of RNAa and the design rules that govern the specificity and sensitivity of dsRNA targeting. 
Restoring E-cadherin expression can reverse EMT and inhibit migration and invasion $(29,30)$. Although, E-cadherin is a well-known tumour suppressor gene, the mechanisms of this inhibition have not been well defined. In this study, the expression of $\beta$-catenin on the surface of the cell membrane was increased via activation of E-cadherin by saRNA, leading to the transfer of $\beta$-catenin from the nucleus to the plasma membrane. With the reduction of $\beta$-catenin in the nucleus, the expression of TCF target genes c-MYC, MMP2 and cyclin D1 was inhibited. $\beta$-catenin has two different cellular functions, namely intercellular adhesion and transcriptional activity. The decrease in cell membrane-bound $\beta$-catenin is associated with the loosening of cell-cell adhesion (31). Normally, E-cadherin and $\beta$-catenin form a complex in the cell-cell junction area, which provides the basis for cell-cell association (32). It has been reported that stabilizing the E-cadherin/ $\beta$-catenin complex can slow EMT and metastasis in colorectal cancer cells (33). The loss of E-cadherin results in the translocation of $\beta$-catenin to the nucleus, where it activates $\beta$-catenin-TCF/LEF-1 target genes and promotes the proliferation and metastasis of cancer (34-36). In the current study, dsEcad-346 and miR-373 inhibited the migration and invasion of $\mathrm{BCa}$ and modulated the expression of E-cadherin/ $\beta$-catenin/TCF target genes. In addition, both saRNAs significantly induced cell cycle arrest and apoptosis.

In summary, a novel dsRNA (dsEcad-346) was designed to increase the expression of E-cadherin. Furthermore, transfection of dsEcad-346 and miR-373 inhibited the growth and metastasis of $\mathrm{BCa}$ cells by promoting redistribution of $\beta$-catenin from nucleus to cell membrane to form the E-cadherin $/ \beta$ catenin complex, and inhibiting transcription of $\beta$-catenin/TCF target genes. The findings demonstrate that dsRNA-mediated upregulation of E-cadherin is an effective strategy to selectively activate the transcription of essential genes. This strategy can be applied to gain-of-function studies and holds great promise as a therapeutic method for BCa treatment.

\section{Acknowledgements}

We sincerely thank the public experimental platform (Tongji Hospital of Huazhong University of Science and Technology, Wuhan, China) for providing experimental facilities.

\section{Funding}

This study was supported by the National Natural Science Foundation of China (grant no. 81372759). The funders had no role in study design, data collection and analysis, decision to publish or preparation of the manuscript.

\section{Availability of data and materials}

The datasets used and/or analysed during the current study are available from the corresponding author on reasonable request.

\section{Authors' contributions}

ZC, CL and JL conceived and designed the experiments. CL and QZ performed the experiments and acquired data. $\mathrm{CL}$ and JL drafted the manuscript. KC contributed to analyzed and interpreted the data. QG and CW critically revised the intellectual content regarding design of dsRNA. All authors have given final approval of the version to be published.

\section{Ethics approval and consent to participate}

Not applicable.

\section{Consent for publication}

Not applicable.

\section{Competing interests}

The authors declare that they have no competing interests.

\section{References}

1. Liang Z, Wang X, Xu X, Xie B, Ji A, Meng S, Li S, Zhu Y, Wu J, $\mathrm{Hu} \mathrm{Z}$, et al: MicroRNA-608 inhibits proliferation of bladder cancer via AKT/FOXO3a signaling pathway. Mol Cancer 16: 96, 2017.

2. Siegel RL, Miller KD and Jemal A: Cancer Statistics, 2017. CA Cancer J Clin 67: 7-30, 2017.

3. Wang S, Liu B, Zhang J, Sun W, Dai C, Sun W and Li Q: Centromere protein $\mathrm{U}$ is a potential target for gene therapy of human bladder cancer. Oncol Rep 38: 735-744, 2017.

4. Kim WJ and Bae SC: Molecular biomarkers in urothelial bladder cancer. Cancer Sci 99: 646-652, 2008.

5. van Roy F: Beyond E-cadherin: Roles of other cadherin superfamily members in cancer. Nat Rev Cancer 14: 121-134, 2014.

6. Breyer J, Gierth M, Shalekenov S, Aziz A, Schäfer J, Burger M, Denzinger S, Hofstädter F, Giedl C and Otto W: Epithelialmesenchymal transformation markers E-cadherin and survivin predict progression of stage pTa urothelial bladder carcinoma. World J Urol 34: 709-716, 2016.

7. Canel M, Serrels A, Frame MC and Brunton VG: E-cadherinintegrin crosstalk in cancer invasion and metastasis. J Cell Sci 126: 393-401, 2013.

8. Das L, Kokate SB, Dixit P, Rath S, Rout N, Singh SP, Crowe SE and Bhattacharyya A: Membrane-bound [beta]-catenin degradation is enhanced by ETS2-mediated Siah1 induction in Helicobacter pylori-infected gastric cancer cells. Oncogenesis 6: e327, 2017.

9. Mukherjee S, Mazumdar M, Chakraborty S, Manna A, Saha S, Khan P, Bhattacharjee P, Guha D, Adhikary A, Mukhjerjee S, et al: Curcumin inhibits breast cancer stem cell migration by amplifying the E-cadherin/ $\beta$-catenin negative feedback loop. Stem Cell Res Ther 5: 116, 2014.

10. Carneiro P, Figueiredo J, Bordeira-Carriço R, Fernandes MS, Carvalho J, Oliveira C and Seruca R: Therapeutic targets associated to E-cadherin dysfunction in gastric cancer. Expert Opin Ther Targets 17: 1187-1201, 2013

11. Wu CL, Ho JY, Chou SC and Yu DS: miR-429 reverses epithelialmesenchymal transition by restoring E-cadherin expression in bladder cancer. Oncotarget 7: 26593-26603, 2016.

12. Lowy AM, Knight J and Groden J: Restoration of E-cadherin/ $\beta$-catenin expression in pancreatic cancer cells inhibits growth by induction of apoptosis. Surgery 132: 141-148, 2002.

13. Li LC, Okino ST, Zhao H, Pookot D, Place RF, Urakami S, Enokida $\mathrm{H}$ and Dahiya R: Small dsRNAs induce transcriptional activation in human cells. Proc Natl Acad Sci USA 103: 17337-17342, 2006.

14. Li C, Ge Q, Liu J, Zhang Q, Wang C, Cui K and Chen Z: Effects of miR-1236-3p and miR-370-5p on activation of $p 21$ in various tumors and its inhibition on the growth of lung cancer cells. Tumour Biol 39: 1010428317710824, 2017.

15. Huang V, Qin Y, Wang J, Wang X, Place RF, Lin G, Lue TF and Li LC: RNAa is conserved in mammalian cells. PLoS One 5: e8848, 2010.

16. Place RF, Li LC, Pookot D, Noonan EJ and Dahiya R: MicroRNA-373 induces expression of genes with complementary promoter sequences. Proc Natl Acad Sci USA 105: 1608-1613, 2008. 
17. Wang J, Place RF, Huang V, Wang X, Noonan EJ, Magyar CE Huang J and Li LC: Prognostic value and function of KLF4 in prostate cancer: RNAa and vector-mediated overexpression identify KLF4 as an inhibitor of tumor cell growth and migration. Cancer Res 70: 10182-10191, 2010.

18. Ting AH, Schuebel KE, Herman JG and Baylin SB: Short doublestranded RNA induces transcriptional gene silencing in human cancer cells in the absence of DNA methylation. Nat Genet 37: 906-910, 2005

19. Livak KJ and Schmittgen TD: Analysis of relative gene expression data using real-time quantitative PCR and the 2(-Delta Delta C(T)) Method. Methods 25: 402-408, 2001.

20. Zhang Q, Wang C, Miao S, Li C, Chen Z and Li F: Enhancing E-cadherin expression via promoter-targeted miR-373 suppresses bladder cancer cells growth and metastasis. Oncotarget 8: 93969-93983, 2017.

21. Junxia W, Ping G, Yuan H, Lijun Z, Jihong R, Fang L, Min L, Xi W, Ting H, Ke D, et al: Double strand RNA-guided endogeneous E-cadherin up-regulation induces the apoptosis and inhibits proliferation of breast carcinoma cells in vitro and in vivo. Cancer Sci 101: 1790-1796, 2010.

22. Xie Y, Li P, Gao Y, Gu L, Chen L, Fan Y, Zhang F and Zhang X: Reduced E-cadherin expression is correlated with poor prognosis in patients with bladder cancer: A systematic review and metaanalysis. Oncotarget 8: 62489-62499, 2017.

23. Kang MR, Li G, Pan T, Xing JC and Li LC: Development of therapeutic dsP21-322 for cancer treatment. Adv Exp Med Biol 983: 217-229, 2017.

24. Weng J, Zhang H, Wang C, Liang J, Chen G, Li W, Tang H and Hou J: miR-373-3p targets DKK1 to promote EMT-induced metastasis via the $\mathrm{Wnt} / \beta$-catenin pathway in tongue squamous cell carcinoma. BioMed Res Int 2017: 6010926, 2017.

25. Lu S, Zhu Q, Zhang Y, Song W, Wilson MJ and Liu P: Dualfunctions of miR-373 and miR-520c by differently regulating the activities of MMP2 and MMP9. J Cell Physiol 230: 1862-1870, 2015.

26. Zhang Y, Zhao FJ, Chen LL, Wang LQ, Nephew KP, Wu YL and Zhang S: miR-373 targeting of the Rab22a oncogene suppresses tumor invasion and metastasis in ovarian cancer. Oncotarget 5: 12291-12303, 2014.

27. Wang J, Place RF, Portnoy V, Huang V, Kang MR, Kosaka M, Ho MKC and Li L-C: Inducing gene expression by targeting promoter sequences using small activating RNAs. J Biol Methods 2: pii: e14, 2015.
28. Janowski BA, Younger ST, Hardy DB, Ram R, Huffman KE and Corey DR: Activating gene expression in mammalian cells with promoter-targeted duplex RNAs. Nat Chem Biol 3: 166-173, 2007.

29. Daugaard I, Sanders KJ, Idica A, Vittayarukskul K, Hamdorf M, Krog JD, Chow R, Jury D, Hansen LL, Hager H, et al: miR-151a induces partial EMT by regulating E-cadherin in NSCLC cells. Oncogenesis 6: e366, 2017.

30. Yuan Z, Wong S, Borrelli A and Chung MA: Down-regulation of MUC1 in cancer cells inhibits cell migration by promoting E-cadherin/catenin complex formation. Biochem Biophys Res Commun 362: 740-746, 2007.

31. Fujita Y, Krause G, Scheffner M, Zechner D, Leddy HEM, Behrens J, Sommer T and Birchmeier W: Hakai, a c-Cbl-like protein, ubiquitinates and induces endocytosis of the E-cadherin complex. Nat Cell Biol 4: 222-231, 2002.

32. Lu Z, Ghosh S, Wang Z and Hunter T: Downregulation of caveolin-1 function by EGF leads to the loss of E-cadherin, increased transcriptional activity of $\beta$-catenin, and enhanced tumor cell invasion. Cancer Cell 4: 499-515, 2003

33. Chen HN, Yuan K, Xie N, Wang K, Huang Z, Chen Y, Dou Q, Wu M, Nice EC, Zhou ZG, et al: PDLIM1 stabilizes the E-cadherin/ $\beta$-catenin complex to prevent epithelial-mesenchymal transition and metastatic potential of colorectal cancer cells. Cancer Res 76: 1122-1134, 2016.

34. Chen L, Zhang A, Li Y, Zhang K, Han L, Du W, Yan W, Li R, Wang Y, Wang K, et al: miR-24 regulates the proliferation and invasion of glioma by ST7L via $\beta$-catenin/Tcf- 4 signaling. Cancer Lett 329: 174-180, 2013.

35. Shin S, Im HJ, Kwon YJ, Ye DJ, Baek HS, Kim D, Choi HK and Chun YJ: Human steroid sulfatase induces Wnt/ $\beta$-catenin signaling and epithelial-mesenchymal transition by upregulating Twistl and HIF-1 $\alpha$ in human prostate and cervical cancer cells. Oncotarget 8: 61604-61617, 2017.

36. Thakur R and Mishra DP: Pharmacological modulation of betacatenin and its applications in cancer therapy. J Cell Mol Med 17: 449-456, 2013.

This work is licensed under a Creative Common Attribution-NonCommercial-NoDerivatives 4.0 International (CC BY-NC-ND 4.0) License. 